Euskal ikerketen aldizkaria | Revue d'études basques |

Revista de estudios vascos | Basque studies review

$16 \mid 2012$

Numéro XVI

\title{
Apport d'un centre de documentation dans la vie d'un laboratoire
}

Jean-Philippe Talec

\section{OpenEdition \\ Journals}

Édition électronique

URL : http://journals.openedition.org/lapurdum/2367

DOI : 10.4000/lapurdum.2367

ISSN : 1965-0655

Éditeur

IKER

Édition imprimée

Date de publication : 1 octobre 2012

Pagination : 117-135

ISSN : 1273-3830

Référence électronique

Jean-Philippe Talec, «Apport d'un centre de documentation dans la vie d'un laboratoire », Lapurdum

[En ligne], 16 | 2012, mis en ligne le 03 décembre 2014, consulté le 20 avril 2019. URL : http://

journals.openedition.org/lapurdum/2367 ; DOI : 10.4000/lapurdum.2367

Talec J.-P. | IKER 


\title{
Apport d'un centre de documentation dans la vie d'un laboratoire de recherche
}

\author{
Jean-Philippe TALEC
}

CNRS-IKER (UMR 5478)

\begin{abstract}
Laburpena: IKER Euskarari eta Euskal Testuei buruzko Ikerketa Gune-ko dokumentazio zentrua ikerketako profesionalen bilera eta mintegi zientifikoak ospatzeko lanerako espazio moduan sortua izan zen. Akitaniako unibertsitateetako (Bordele 3, UPPA) liburutegiei loturik, $100 \mathrm{~m}^{2}$ baino gehiago eta 5000 dokumentutik gora (1809 liburu, euskal ikerketari eskainiriko aldizkari, tesiak, memoriak, eta ikasleen lanak) eskaintzen ditu. Euskal ikasketen testuinguruan informaziorako eta formakuntzarako sostengu garrantzitsu bat da dokumentazio zentrua. Horretaz gain, zentrua ikerketa proiektuetan biltzen diren datuen artxibategi moduan ere erabiltzen da. Azkenik, Lapurdum euskal ikasketen aldizkariaren zenbakiak Revues.org plataformako Access Gold delakoan eskuragarri jartzeaz arduratzeaz gain, laborategiko gaurkotasun zientifikoak hedatzeaz ere arduratzen da.
\end{abstract}

\section{Présentation générale}

« La constitution de grammaires et de dictionnaires est d'une utilité fondamentale pour les chercheurs comme pour les locuteurs des langues. Cette documentation est la base indispensable des recherches typologiques et comparatives. Elle permet d'enregistrer des états de langue, clé essentielle pour leur comparaison et pour la compréhension de leur évolution. Les dictionnaires mettent à la disposition des chercheurs des bases lexicales indispensables à toute entreprise de comparatisme historique et de reconstitution du vocabulaire de base commun à une famille donnée. » (BOËTSCH 2011 : 642)

Les études sur les langues régionales ne pouvaient pas se développer sans bibliothèque de recherche. Depuis 1999, les directeurs successifs de l'Unité Mixte de Recherche (UMR) 5478 IKER $^{1}$ sur la langue et les textes basques avaient bien compris l'intérêt de constituer une

1.- Depuis sa création, l'UMR a connu deux directions successives, celle de Bernard Oyharçabal (Directeur de recherche CNRS) de 1999 à 2009, puis celle de Ricardo Etxepare (Chargé de recherche CNRS) de 2009 à aujourd'hui. 
collection de documents très spécialisés pour soutenir leurs activités. Aussi pendant dix ans, les chercheurs de l'unité ont créé un fonds documentaire et l'ont augmenté avec des achats de monographies en linguistique et littérature et des donations de livres et de revues ayant appartenu au Professeur d'université René Lafon (1899-1974) et au chanoine Pierre Lafitte (1901-1985). En 2009 trois changements importants permirent au centre de documentation du laboratoire d'ouvrir ses portes aux lecteurs; (i) l'achèvement des travaux de rénovation des locaux du Château-Neuf mis en oeuvre par la Communauté d'Agglomération de BayonneAnglet-Biarritz (CABAB) dans le cadre de la mise à disposition de l'UPPA de ce bâtiment appartenant à la ville de Bayonne, (ii) le déménagement de l'UMR vers ces locaux situés sur le campus de la Nive, et (iii) la livraison des matériels réseaux, informatiques et mobiliers notifiés dans les marchés publics de "Fourniture d'équipements pour les nouveaux locaux de l'Université de Pau et des Pays de l'Adour (UPPA) sur le site de la Nive "».

Après cette complète réorganisation, où en est le jeune centre de documentation IKER? Quelle place occupe t-il dans le laboratoire de recherche? Dans quelles directions sont tournés les services documentaires? Sont-ils adaptés aux chercheurs du Centre National de la Recherche Scientifique (CNRS), de l'enseignement supérieur aquitain (Michel de Montaigne - Bordeaux 3 et UPPA) et aux masters du Département interuniversitaire d'Etudes Basques (DIEB) ${ }^{4}$ ? Les apports de la documentation de l'unité complètent-elles les autres fonds documentaires du

Pays basque français dans lesquels on compterait plus de 70.000 documents $^{5}$ ?

Cette communication présente les actions récentes du centre de documentation avec des données thématiques et volumétriques sur les ressources documentaires de l'unité IKER de 2009 à 2012. Nous résumerons ce qui nous semble essentiel dans cinq chapitres distincts ; (i) la création du centre de documentation, (ii) la présentation des imprimés conservés dans nos locaux, (iii) le signalement des documents dans un catalogue informatisé dont le nom de code est Kutxa (avec le logiciel libre Koha ${ }^{6}$ ), (iv) les ressources documentaires en ligne accessibles via les partenaires de l'UMR, (v) enfin un aperçu de la diffusion des savoirs en texte intégral de l'unité avec le soutien du Centre pour la Communication Scientifique Directe (CCSD) et du Centre pour l'édition électronique ouverte (Cléo).

\subsection{Contexte de la création du centre de documentation}

Installé sur une surface de $100 \mathrm{~m}^{2}$ au cœur du Château-Neuf, l'espace documentaire

2.- Documentation. IKER UMR5478 (CNRS, Bordeaux 3, UPPA). À propos du centre de documentation [en ligne]. Disponible sur : <http://www.iker.cnrs.fr/-centre-de-documentation-.html> (consulté le ler septembre 2012)

3.- Campus de la Nive : histoire d'une mutation [en ligne]. Disponible sur <http://www.univ-pau.fr/live/ digitalAssets/66/66672_Histoire_Nive.pdf> (consulté le ler septembre 2012)

4.- Présentation du DIEB [en ligne]. Disponible sur < http://ufr-pluribab.univ-pau.fr/live/licence/licence-etudesbasques> (consulté le ler septembre 2012)

5.- Une enquête menée par la Médiathèque de Bayonne révélait que «les fonds repérés se recouvrent certainement dans une proportion importante, à mesurer. On peut estimer que le fonds basque représente aujourd'hui au moins 40.000 monographies, 1.000 à 1.500 titres de périodiques, 30.000 documents iconographiques, des centaines de manuscrits ». (BLIN \& OURET 2011)

6.- KohaLa. Koha-fr : communauté francophone koha [en ligne]. Disponible sur : <http://www.koha-fr.org> (consulté le ler septembre 2012) 
donne accès à une vingtaine de places assises, une borne wifi, deux postes informatiques et plus de 5.000 documents publiés de 1809 jusqu'à nos jours. En très grande majorité il s'agit de livres et périodiques sur le Pays basque, la linguistique et la littérature dont le niveau de lectorat est égal ou supérieur au niveau doctorat. Quelques manuscrits additionnels, d'élèves de l'ancien Petit Séminaire Saint-François-Xavier d'Ustaritz, sont conservés à des fins de recherche tout comme d'anciens dictionnaires relatifs aux dialectes labourdin, bas-navarrais et souletin ${ }^{7}$. Nous ne détenons pas de document iconographique (estampe, gravure, affiche, photographie) et d'enregistrement audiovisuel. Par contre nous proposons aux membres de la communauté universitaire de nombreuses bases de données scientifiques en sciences humaines et sociales (SHS) ${ }^{8}$.

Depuis la fin d'année 2010 le centre de documentation est associé au service commun de documentation (SCD) de l'université Michel de Montaigne - Bordeaux $3^{9}$ et au cours des dernières années il a bénéficié du soutien du SCD de l'UPPA ${ }^{10}$ et des services en ligne de la bibliothèque $\mathrm{HABE}^{11}$, spécialisée sur le plurilinguisme et dépendante du Gouvernement autonome basque (Eusko Jaurlaritza). Il a l'obligation « d'informer le SCD associé des crédits documentaires et de leur utilisation ${ }^{12} »$. Un Conseil de documentation se réunit deux fois par an. Il regroupe le directeur de l'UMR, trois chercheurs et enseignants-chercheurs, lesquels représentent toutes les tutelles du laboratoire, et le responsable des collections documentaires chargé ensuite des traitements. Ils fixent ensemble la politique documentaire et les conditions pratiques de fonctionnement.

Le centre de documentation accueille les chercheurs, enseignants-chercheurs et doctorants sans interruption pendant l'année universitaire. Pour les autres étudiants avancés, masters Recherche et Enseignement du DIEB, la salle de documentation est ouverte du lundi au vendredi les après-midi. Nous proposons divers services allant du renseignement bibliographique à la valorisation des productions scientifiques du laboratoire en passant par le prêt à domicile.

7.- Documentation. IKER UMR5478 (CNRS, Bordeaux 3, UPPA). Page d'information sur les dialectes basques dans le catalogue documentaire Kutxa [en ligne]. Disponible sur : <http://iker-koha.univ-pau.fr/cgi-bin/ koha/opac-search.pl?idx=ti\&q=dialecte> (consulté le ler septembre 2012)

8.- Academic Search Premier, Arts \& Humanities Citation Index, Conference Proceedings, Dissertation \& Theses, MLA International Bibliography ou encore ProQuest Dissertations and Theses, fournissent aux chercheurs académiques plus de 10.000 publications en texte intégral.

9.- SCD. Université Bordeaux 3. Accueil Service Commun de Documentation de l'Université Bordeaux 3 [en ligne]. Disponible sur : <http://www.u-bordeaux3.fr/fr/documentation.html> (consulté le ler septembre 2012)

10.- SCD. Université de Pau et des Pays de l'Adour (UPPA). Accueil Service Commun de Documentation [en ligne]. Disponible sur : <http://bibliotheques.univ-pau.fr/live> (consulté le ler septembre 2012)

11.- HABE IKASBIL. HABE Liburutegia [en ligne]. Disponible sur : <http://www.ikasbil.net/web/liburutegia/ home> (consulté le ler septembre 2012)

12.- Décret n² 2011-996 du 23 août 2011 relatif aux bibliothèques et autres structures de documentation des établissements d'enseignement supérieur créées sous forme de services communs, article 9. 


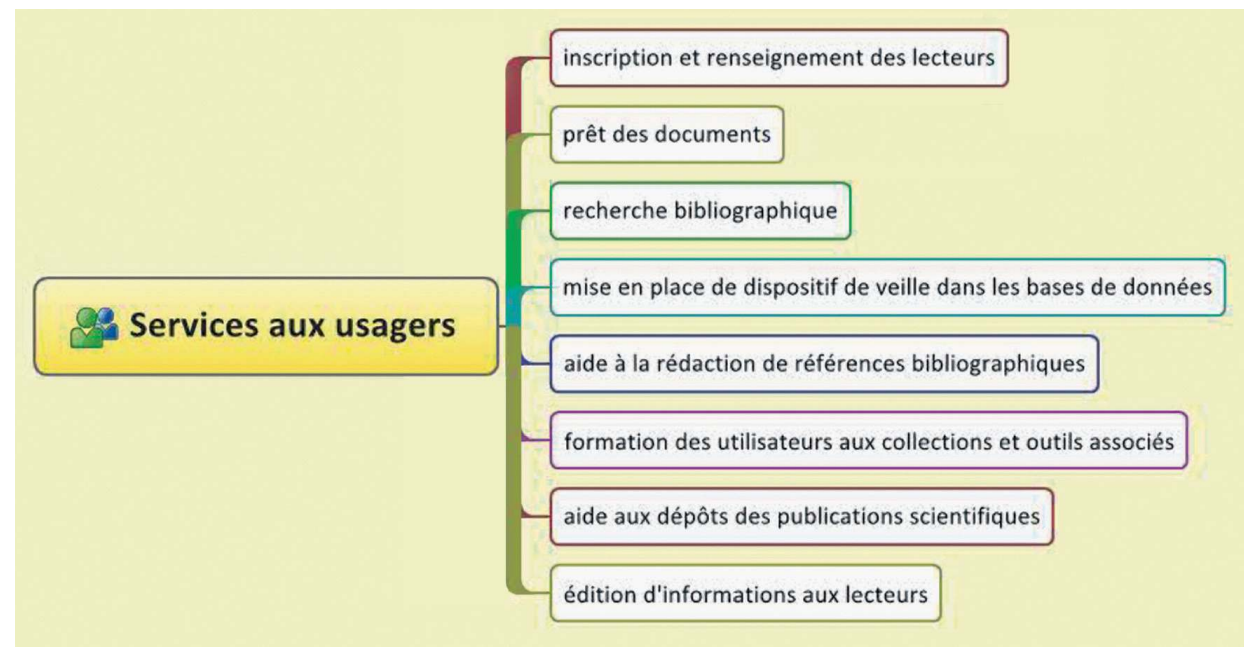

Figure 1 : Services documentaires orientés vers les usagers

Les règles de circulation des documents sont fixées dans le règlement intérieur. Les chercheurs non affiliés à un établissement universitaire peuvent demander l'accès au centre de documentation. La démarche à suivre est de soumettre un court projet de recherche à la direction du laboratoire afin d'examiner sa faisabilité. Le dossier doit détailler quels sont les objectifs et les résultats attendus par le candidat, ainsi que la nature des documents et des équipements recherchés. À partir du moment où la proposition de projet est envoyée au centre de recherche, les délais de réponse sont généralement de moins de trois semaines.

Tableau 1 : Conditions de prêt de documents

\begin{tabular}{ll}
\hline Public & Nombre, durée \\
\hline Chercheur, enseignant-chercheur & 20 documents, 60 jours \\
Ingénieur, technicien, administratif & 20 documents, 60 jours \\
Personnel des projets de recherche & 20 documents, 60 jours \\
Doctorant & 10 documents, 60 jours \\
Master & 10 documents, 30 jours \\
\hline
\end{tabular}

\subsection{Collections revues et augmentées}

Nous conservons à parts presque égales des monographies (ouvrages, thèses, mémoires universitaires) et des revues consacrées aux domaines de la civilisation basque. Les collections imprimées sont disposées dans la salle de documentation ( $72 \%$ des documents) et plus rarement dans des réserves. En très grande majorité les publications couvrent la période du $16^{e}$ siècle à nos jours. Louvrage le plus ancien du fonds documentaire fut écrit par Martin Duhalde 
(1733?-1804) ${ }^{13}$ et édité après sa mort en 1809. Il s'intitule « Meditacioneac gei premiatsuenen gainean: Cembait abisuekin, Othoitcekin eta bicitceco Erregela batekin $»^{14}$. Il s'agit d'un livre de méditations et prières imprimé à Bayonne chez Cluzeau. La dernière acquisition était un recueil de communications scientifiques données à Kyoto dans une conférence sur l'évolution $\mathrm{du}$ langage "The evolution of language : proceedings of the 9th International Conference (EVOLANG9) " sous la direction de Thomas C Scott-Phillips, Monica Tamariz, Erica A Cartmill et James R Hurford, et édité chez World Scientific Publishing en 2012.

Au fil du temps, nous avons rassemblé une collection multilingue de plus 2.800 monographies ${ }^{15}$ avec des titres ${ }^{16}$ en langues basque, française, espagnole et anglaise. Ils sont organisés par disciplines (philosophie, religion, sciences sociales, langues, littératures etc.) et disponibles en libre accès dans la salle de documentation.

Tableau 2 : Disciplines réprésentées en sciences humaines et sociales

\begin{tabular}{|l|l|}
\hline Discipline & Matières \\
\hline Linguistique & $\begin{array}{l}\text { Grammaire, sémantique, dialectologie, } \\
\text { phonétique, lexicographie, variation linguistique, } \\
\text { contact de langues, code switching, atlas } \\
\text { linguistique }\end{array}$ \\
\hline Littérature & $\begin{array}{l}\text { Critique littéraire, littérature comparée, génétique } \\
\text { des textes, conte, légende }\end{array}$ \\
\hline Histoire & $\begin{array}{l}\text { Histoire classique, moderne et contemporaine } \\
\text { Géographie }\end{array}$ \\
\hline \\
toponymie de voyages, géographie historique,
\end{tabular}

13.- URQUIJO IBARRA, Julio de. Notas de Bibliografía vasca. 4, ¿ De qué obra francesa tradujo Duhalde sus Meditaciones ? Revista Internacional de los Estudios Vascos = Revue Internationale des Etudes Basques. 1908, no 2. pp. 313-323. [en ligne]. Disponible sur : <http://hedatuz.euskomedia.org/4768/1/02313323. pdf> (consulté le ler septembre 2012)

14.- Lassociation de promotion de la littérature basque ZABALIK (basée à Saint-Palais) a donné au centre de documentation l'ouvrage cité ainsi que d'autres livres de dévotion et bibles du $19^{\text {e }}$ siècle. Par ailleurs Zablik conserve le fonds personnel de l'écrivain et moine franciscain Manex Erdozaintzi-Etxart (1934-1984).

15.- Il y a des publications de nombreux linguistes ayant travaillé sur l'histoire de la langue basque par exemple Jacques Allières, Luca Cavalli-Sforza, Jean-Baptiste Coyos, Ricardo Gómez, Jean Haritschelhar, Pierre Lafitte, Pierre Lhande, Luis Michelena, Jean-Baptiste Orpustan, Bernard Oyharçabal, Georges Rebuschi, Lawrence Trask, Charles Videgain, Koldo Zuazo...

16.-Plus de 1.000 noms de personnes sont référencés dans la base de données Kutxa. Ils figurent dans les publications le plus souvent en tant qu'auteur, préfacier, directeur de thèse, directeur de la publication ou éditeur scientifique. 
Sociologie, anthropologie, ethnologie, tradition, mœurs et coutumes, organisation des systèmes éducatifs, enseignement supérieur, didactique des langues, sciences politiques

Sciences sociales langues, sciences politiques

Musique, théâtre, sport et loisirs

Pastorale, bertsolarisme, chanson populaire, danse, pelote

Sciences de l'information

Bibliothèque, histoire du livre, édition, bibliographie

Philosophie, religion

Bibles et livres de dévotion traduits en basque

Tableau 3 : Répartition des monographies par langue (2012)

\begin{tabular}{|l|c|}
\hline Langue & Répartition \\
\hline Basque & $32 \%$ \\
\hline Français & $26 \%$ \\
\hline Anglais & $20 \%$ \\
\hline Espagnol & $19 \%$ \\
\hline $\begin{array}{l}\text { Autres langues occitano romanes (catalan, corse, italien, } \\
\text { latin, occitan, portugais) }\end{array}$ & $1 \%$ \\
\hline Autres langues germaniques (allemand, néerlandais) & $1 \%$ \\
\hline Autres langues (langues celtiques, sémitiques, kartvéliennes) & $1 \%$ \\
\hline
\end{tabular}

Dans le numéro XII de la revue Lapurdum ${ }^{17}$, Jean-Claude Poitelon, ancien chef de service à la Bibliothèque nationale de France, estimait à 1.300 les journaux, revues, bulletins et magazines parues au Pays basque français entre 1944 et 2004 :

«Au terme de ces longues recherches, nombreuses et parfois obstinées, et grâce à la collaboration de tant de personnes, ont pu être rassemblés des centaines de titres et des milliers de fascicules, versés au fur et à mesure à la Bibliothèque nationale de France, dont les collections ont été considérablement enrichies. La bibliographie qui suit atteste l'importance des résultats; elle décrit quelque 1375 périodiques basques parus en France de

17.- IKER UMR5478 (CNRS, Bordeaux 3, UPPA). Lapurdum - Euskal ikerketen aldizkaria : Revue d'études basques [en ligne]. Disponible sur : <http://lapurdum.revues.org> (consulté le ler septembre 2012) 
1944 à 2004, représentés maintenant à 90 \% dans les collections nationales. Jusque-là aucune bibliothèque n'en regroupait guère plus de 500. » (POITELON 2008: 7)

Ce total montre la richesse des publications en série régionales dont la première rédigée intégralement en langue basque remonte à Bayonne en 1848 avec l'Uscal-Herrico Gaseta (1848-1852), supplément du journal politique Ariel (CAILLOT 2002 : 24 p.) fondé par Augustin Chaho (1811-1858).

En matière de périodique nous proposons 70 titres, ce qui représente 2.600 exemplaires. Un tiers des numéros provient du Musée basque et de l'histoire de Bayonne ${ }^{18}$ et de doublons donnés à l'UMR juste après son transfert au Château-Neuf. Certains titres ont commencé à être publiés au début du XXe siècle ${ }^{19}$ ou entre les deux guerres ${ }^{20}$. La plupart des revues conservées sur place ${ }^{21}$ sont plus récentes et éditées par des institutions universitaires ${ }^{22}$. Il n'est pas facile d'évoquer ici les dizaines de milliers articles parus depuis un siècle. La liste de ces textes, dont les sujets abordent l'ethnologie, l'histoire de la langue, les marins et la pêche maritime, les critiques littéraires, les écrivains basques d'aujourd'hui, serait trop longue.

Le dernier inventaire montrait une continuité dans l'accroissement des collections. Lannée dernière, 310 nouveaux documents avaient rejoint le fonds documentaire. Il s'agissait de livres achetées à des éditeurs spécialisés en SHS (une centaine), plus divers documents obtenus par des dons (livres anciens, tirés à part) et échanges (exemplaires de revues d'études basques).

Tableau 4 : Collections documentaires en unité (2012)

\begin{tabular}{|l|r|r|r|}
\hline Type de document & 2009-2010 & 2010-2011 & 2011-2012 \\
\hline Livres, thèses, mémoires & 2.400 & 2.660 & 2.870 \\
\hline Livres anciens (19e siècle) & 60 & 60 & 60 \\
\hline Monographies (20 siècle) & 1.340 & 1.420 & 1.490 \\
\hline Monographies (21 $1^{\mathrm{e}}$ siècle) & 1.000 & 1.180 & 1.310 \\
\hline Périodiques imprimés & $\begin{array}{r}70 \text { titres } \\
\text { 2.500 volumes }\end{array}$ & $\begin{array}{r}70 \text { titres } \\
2.550 \text { volumes }\end{array}$ & $\begin{array}{r}70 \text { titres } \\
2.600 \text { volumes }\end{array}$ \\
\hline
\end{tabular}

18.- Musée Basque et de l'histoire de Bayonne. Bibliothèque et centre de documentation [en ligne]. Disponible sur : <http://www.museebasque.com/fr/documentation> (consulté le ler septembre 2012)

19.- Revista internacional de los estudios vascos (RIEV, 1907-), Euskalerriaren alde (1911-1931)

20.- Gure herria (1921-1976), Bulletin du Musée Basque (1924-)

21.- Boletín de la Real Sociedad Bascongada de los Amigos del País (1945-), Jakin (1956-), Fontes linguae vasconum (1969-), Maiatz (1982), Hegats (1989-)...

22.- Anuario del Seminario de Filología Vasca «Julio de Urquijo » (1954-), Bat soziolinguistika aldizkaria : hizkuntz normalkuntza eta glotopolitika aldizkaria (1990-), Gogoa (2001), Iura vasconiae (2004-), Mundaiz (1975-), Revista de lenguas y literaturas catalana, gallega y vasca (1991-), Uztaro (1990-)... 


\begin{tabular}{|l|r|r|r|}
\hline Manuscrits & une centaine & une centaine & une centaine \\
\hline Articles, tirés à part, autres & 300 & 350 & 400 \\
\hline Accroissement annuel & 390 & 360 & 310 \\
\hline
\end{tabular}

\subsection{Signalement des documents dans un catalogue en ligne}

Le centre de documentation réalisait la première informatisation du fonds avec l'appui technique du Centre de ressources informatiques (CRI ${ }^{23}$ de l'UPPA en 2007. Nous avons choisi d'utiliser le système intégré de gestion de bibliothèque (SIGB) Open Source Koha (ENGARD 2010) qui disposait de la plupart des fonctionnalités de gestion d'une bibliothèque de recherche (acquisition, catalogage, circulation, catalogue Internet). Consultable en ligne, le catalogue de la documentation IKER se nomme Kutxa ${ }^{24}$. Celui-ci identifie les textes conservés au laboratoire de manière précise (ISBN, ISSN, auteur, titre, collection, date et lieu d'édition) quel que soit le type de document (livre, périodique, thèse, mémoire universitaire, ressource électronique). C'est un outil de recherche de nouvelle génération qui fonctionne selon les standards du web 2.0 (YANG \& HOFMANN 2010). Les chercheurs peuvent se connecter au serveur et réserver à distance un ou plusieurs documents du centre. À l'heure actuelle le Kutxa compte 2.400 notices documentaires normalisées aux formats bibliographiques historiques UNIversal MARC (UNIMARC) et International Standard Bibliographic Description (ISBD). 400 nouvelles notices sont créées en moyenne par an depuis le début de l'informatisation du fonds. Au terme « euskara », le moteur de recherche intégré 25 signalera plus de 170 réponses. Ce sont des enquêtes sociolinguistiques, grammaires et atlas linguistiques que l'on ne trouve pas facilement sur Bayonne. Des liens hypertextes affinent les sélections par disponibilité, auteur(s), collection(s) et sujets du document et orientent aussi l'utilisateur vers des mémoires universitaires ou ebooks en texte intégral. Loption " parcourir par hiérarchie » est basée sur la Classification décimale de Melvil Dewey (1851-1931). Elle répartit les notices du catalogue en fonction des sujets représentés (archéologie, histoire, géographie, langues, littérature...). Si la page web affiche une imagette de la première de couverture alors cela signifie que la notice bibliographique est enrichie par une référence de la plateforme Google Book où se trouvent des compléments comme le sommaire de l'ouvrage.

En 2012, le catalogue Kutxa avait reçu 8.816 visites et affiché 72.723 pages. Les statistiques du site révélaient que certains visiteurs suivaient les dernières acquisitions à travers les fils RSS et récupéraient les données dans différents formats bibliographiques ${ }^{26}$.

23.- Centre de ressources informatiques (CRI). Université de Pau et des Pays de l'Adour (UPPA). [en ligne]. Disponible sur : <http://cri.univ-pau.fr/live> (consulté le ler septembre 2012)

24.- Documentation. IKER UMR 5478 (CNRS, Bordeaux 3, UPPA). Catalogue kutxa [en ligne]. Disponible sur: $<$ http://iker-koha.univ-pau.fr> (consulté le ler septembre 2012)

25.- Koha utilise le moteur d'indexation et de recherche Zebra adapté pour les catalogues de plus de 10.000 notices bibliographiques. C'est un outil capable de lire les enregistrements dans différents formats d'entrée (XML, MARC). Il donne accès aux données grâce à des équations de recherche booléenne et des requêtes en texte libre.

26.- BIBTEX, RIS, Bublin Core, MARC 
Tableau 5 : Fonctionnalités du système de gestion de bibliothèque Koha

\begin{tabular}{|c|c|}
\hline Fonctionnalité & Remarque \\
\hline $\begin{array}{l}\text { Catalogue public } \\
\text { web }\end{array}$ & $\begin{array}{l}\text { Il n'est pas nécessaire d'installer un logiciel particulier sur } \\
\text { l'ordinateur client. Un navigateur web suffit pour accéder au } \\
\text { catalogue. }\end{array}$ \\
\hline $\begin{array}{l}\text { Interface } \\
\text { multilingue }\end{array}$ & $\begin{array}{l}\text { Koha donne aux chercheurs le choix d'interroger le catalogue en } \\
\text { français, anglais et espagnol. }\end{array}$ \\
\hline Compte lecteur & $\begin{array}{l}\text { Une partie privée fournit un historique des prêts à l'adhérent, elle } \\
\text { lui permet de prolonger ceux-ci si nécessaire, d'annoter les notices } \\
\text { documentaires avec des tags ou de créer des listes personnalisées } \\
\text { de référence bibliographique. }\end{array}$ \\
\hline $\begin{array}{l}\text { Export } \\
\text { bibliographique }\end{array}$ & $\begin{array}{l}\text { Koha facilite l'export des références dans des logiciels } \\
\text { bibliographiques. }\end{array}$ \\
\hline $\begin{array}{l}\text { Contenus } \\
\text { enrichis }\end{array}$ & $\begin{array}{l}\text { Le catalogue importe des données provenant de Google Book, par } \\
\text { exemple des vignettes d'ouvrages. }\end{array}$ \\
\hline $\begin{array}{l}\text { Recherche par } \\
\text { mots-clés et } \\
\text { expressions }\end{array}$ & $\begin{array}{l}\text { Le moteur Zebra autorise les recherches sur un champ de la base de } \\
\text { données (auteur, titre, sujet) ou sur l'ensemble de la notice avec des } \\
\text { opérateurs booléens et des expressions exactes. Les résultats sont } \\
\text { triés ensuite par popularité, auteur, cote, date ou titre. }\end{array}$ \\
\hline $\begin{array}{l}\text { Recherche par } \\
\text { navigation }\end{array}$ & $\begin{array}{l}\text { Les visiteurs naviguent dans les thèmes et les sous thèmes du } \\
\text { catalogue à partir de l'option « Parcourir par hiérarchie ». }\end{array}$ \\
\hline $\begin{array}{l}\text { Abonnement à } \\
\text { des flux RSS }\end{array}$ & $\begin{array}{l}\text { Koha affiche le nombre de résultats obtenus dans la recherche. } \\
\text { Licône orange à côté des résultats donne les moyens aux lecteurs } \\
\text { de s'abonner au fil RSS associé à la recherche. }\end{array}$ \\
\hline
\end{tabular}

\subsection{Ressources numériques des partenaires documentaires}

Le numérique a radicalement changé les modes de diffusion des connaissances sans pour autant réduire le coût de l'information scientifique et technique. Les bibliothèques de recherche accomplissent leurs acquisitions dans un contexte où les prix des publications scientifiques augmentent chaque année. L'application du nouveau taux de TVA à $7 \%$ sur le livre imprimé dès le $1^{\text {er }}$ avril 2012 ne devrait pas arrêter cette dynamique.

«En France, entre 1980 et 1999, le coût des périodiques étrangers acquis par les bibliothèques universitaires a progressé de $229 \%$ pour les lettres et les sciences humaines, et de plus de $600 \%$ pour la pharmacie. De 2000 à 2007, ce taux d'augmentation a diminué mais il a tout 
de même atteint 50 \% pour les mêmes périodiques étrangers. Aujourd'hui, dans un contexte de crise économique et financière, les bouquets d'abonnements électroniques augmentent en moyenne d'environ 5 \% par an. » (RENOULT 2011)

Par conséquent il est essentiel d'avoir une approche pragmatique de la documentation en s'assurant que d'abord son besoin ne se trouve pas dans les achats électroniques déjà négociées par le CNRS et les universités tutelles auprès des éditeurs et diffuseurs scientifiques. Il convient ensuite d'intégrer les acquisitions dans les projets de recherche ou de chercher de nouveaux partenariats avec des bibliothèques spécialisées du Pays basque (AGIRREAZALDEGI BERRIOZABAL 2011).

\subsection{Des dizaines de milliers de documents scientifiques en ligne}

Une grande partie des abonnements numériques du laboratoire dépend des droits acquis auprès des éditeurs commerciaux scientifiques (Elsevier Science et Thomson Reuters) par le CNRS et les universités Bordeaux 3 et UPPA ${ }^{27}$. Cette offre en ligne se matérialise par des bases de données bibliographiques, des articles de revues en texte intégral et des livres électroniques (ebooks). Par exemple nous sommes abonnés aux produits et services en ligne des bases Academic Search Premier, Web of Science, Modern Language Association (MLA), FRANCIS, JSTOR, CAIRN dans le domaine de la linguistique et des lettres. Par ailleurs l'Institut public $\mathrm{HABE}^{28}$ a signé une convention avec le laboratoire IKER et trois autres acteurs de l'enseignement supérieur et de la recherche Soziolinguistika Klusterra, Mintzola Fundazioa et la Fundación Asmoz favorisant l'utilisation de ressources documentaires dans les domaines du multilinguisme et de l'enseignement des langues. Laccord passé à Donostia-Saint-Sébastien le 16 décembre 2011 assure au personnel d'IKER la fourniture de documents scientifiques supplémentaires par voie électronique.

Une enquête sur les pratiques des utilisateurs du centre de documentation réalisée en juin $2011^{29}$ montrait que $50 \%$ des chercheurs de l'UMR consultaient régulièrement BiblioSHS ${ }^{30}$. Le service de documentation organise des ateliers (individualisés ou en groupe) dans lesquels sont précisés le mode de fonctionnement des plateformes d'information scientifique et technique, des moteurs de recherche et des logiciels de bibliographie (JabRef, Zotero). Enfin le centre de documentation a mis en œuvre un serveur de fichiers sécurisé (projet OPARO) avec le CRI de l'UPPA qui permet de partager des données électroniques à travers un réseau. Après un an de service nous constatons que les chercheurs l'utilisaient essentiellement pour conserver des

27.- BiblioSHS propose plus de 10.000 publications gratuites en texte intégral. Dans les ressources numériques des SCD de l'UPPA et de Bordeaux 3 il y a des abonnements complémentaires en linguistique comme les numéros des revues Annual Review of Applied Linguistics, Journal of the International Phonetic Association, Language in Society, Phonology Cambridge, Linguistics, Multilingua, Probus, The Linguistic Review, Theoretical Linguistics...

28.- La bibliothèque HABE dispose d'environ 40.000 documents et 650 titres de revue.

29.- TALEC, J.-P. Enquête sur les pratiques informationnelles de l'UMR 5478 IKER. Bayonne, 2011. 6 p.

30.- INIST-CNRS. Présentation du portail BiblioSHS : portail d'information scientifique des unités CNRS en Sciences humaines et sociales [en ligne]. Disponible sur : <http://biblioshs.inist.fr/sites/biblioshs/IMG/ppt/ TutorielBBSHSV3.pps> (consulté le ler septembre 2012) 
publications scientifiques et des données produites pendant les projets de recherche ${ }^{31}$.

\section{Contributions au libres accès}

Le laboratoire contribue au libre accès à travers une archive ouverte et une revue électronique. Il met à disposition des résultats de la recherche au plus grand nombre sans restriction d'accès dans le but d'accélérer la diffusion des résultats de recherche.

\subsection{Une archive ouverte HAL thématique : Artxiker}

Avant la création du centre de documentation le laboratoire de recherche IKER s'était engagé dans le mouvement du libre accès à l'information scientifique en 2006 à travers l'archive ouverte Artxiker ${ }^{32}$ mise en service par le Centre pour la Communication Scientifique Directe (CCSD) sur le serveur Internet Hyper Articles en Ligne (HAL). Il s'agit d'une plateforme qui permet aux chercheurs d'y déposer leurs publications pour une consultation libre par tout utilisateur d'Internet. Cette archive fournit des productions intellectuelles de la recherche internationale dans le domaine de la langue basque et des langues typologiquement proches. Le dépôt des archives est géré par Jean-Baptiste Coyos, membre honoraire du centre IKER, lequel incite les auteurs de la recherche et de l'enseignement supérieur à diffuser leurs travaux pour être lus, téléchargés et imprimés. La propriété intellectuelle des documents déposés appartient à leur(s) auteur(s). En 2006 J.-B. Coyos, revenait sur le contexte singulier des publications scientifiques en basque :

\footnotetext{
«Si la recherche sur la langue basque suscite toujours beaucoup d'intérêt et attire l'attention de nombreux chercheurs à travers le monde, il faut savoir que la documentation scientifique dont elle fait l'objet est particulièrement dispersée. En effet, les revues spécialisées sont publiées en Pays basque et ont relativement peu d'audience à l'extérieur, beaucoup d'articles étant rédigés en langue basque. Les résultats de nombreux travaux restent peu diffusés, s'ils ne sont pas publiés dans l'une des langues des publications internationales. Larchive électronique Artxiker souhaite devenir le lieu passerelle d'échange des recherches de la communauté mondiale des bascologues, des spécialistes des langues ergatives, plus généralement des typologues amenés à étudier la langue basque ou des langues proches. » (COYOS 2010 : 343)
}

En 2012 Artxiker totalisait 360 dépôts. La plupart concernait des articles publiés dans des revues, des communications avec actes, des chapitres d'ouvrages scientifiques, des rapports de recherche et des thèses. Les travaux scientifiques portaient sur la morphosyntaxe, la phonologie, la sémantique, la lexicologie et l'histoire de la langue basque.

31.- Les informations d'OPARO sont accessibles aux membres de l'UMR sur les stations informatiques du Château-Neuf et via un Réseau privé virtuel (VPN).

32.- Artxiker: Archive de la Recherche pour la Langue basque et les Langues typologiquement proches [en ligne]. Bayonne, IKER UMR 5478 (CNRS, Bordeaux 3, UPPA), CCSD (Centre pour la Communication Scientifique Directe - UPS2275), Disponible sur : <http://artxiker.ccsd.cnrs.fr> (consulté le ler septembre 2012). 


\subsection{Une revue d'études basques sur le portail Revues.org : Lapurdum}

En 2008, la direction de la revue d'études basques Lapurdum et le Centre pour l'édition électronique ouverte (Cléo) du CNRS signait une convention dans laquelle était stipulée la volonté de publier le maximum d'articles en texte intégral sur le portail de revues en sciences humaines et sociales Revues.org ${ }^{33}$. Il semblerait que Lapurdum soit devenu à cette occasion la première revue produite en Aquitaine diffusée sur le portail Revues.org. Le centre de documentation a placé l'intégralité du corpus Lapurdum (1996-) en ligne avec le logiciel Lode $^{34}$. Aujourd'hui la collection est complète, avec 13 numéros, dont deux spéciaux et elle comporte plus de 300 articles écrits par une centaine d'auteurs. Tous les articles se présentent de manière identique dans les fichiers Extensible HyperText Markup Language (XHTML) avec d'abord une partie métadonnées (titre, sous-titre, auteur, pagination) puis une barre d'accès rapide aux éléments de la page web (résumé, index, plan, texte, bibliographie, annexes, notes, auteur). Ils sont téléchargeables au format Portable Document Format (PDF) sans aucune restriction particulière. Pour aider la recherche documentaire il existe des index auteurs et matières (sujets, géographie et chronologie) et un moteur de recherche. À droite de la page d'accueil des flux d'actualité RSS, fournis par l'album des sciences sociales Calenda ${ }^{35}$ et le site de l'UMR, sont affichés et proposés sous forme d'abonnement.

Le site de la revue Lapurdum a augmenté de plus de $50 \%$ sa fréquentation en 2012 par rapport à l'année précédente. Nous avons constaté que les visites étaient passées de 51.912 en 2011 à 90.103 en 2012. Au cours des douze derniers mois 225.623 pages avaient été demandées par des utilisateurs le plus souvent connectés en France, Espagne, Belgique, EtatsUnis et Canada.

33.- Centre pour l'édition électronique ouverte (Cléo) du CNRS. Revues.org - Portail de revues en sciences humaines et sociales [en ligne]. Disponible sur : <http://www.revues.org> (consulté le ler septembre 2012)

34.- Centre pour l'édition électronique ouverte (Cléo) du CNRS. Lodel, logiciel d'édition électronique [en ligne]. Disponible sur : <http://www.lodel.org> (consulté le ler septembre 2012)

35.- Centre pour l'édition électronique ouverte (Cléo) du CNRS. Calenda : Calendrier des sciences humaines et sociales [en ligne]. Disponible sur : <http://calenda.revues.org> (consulté le ler septembre 2012) 


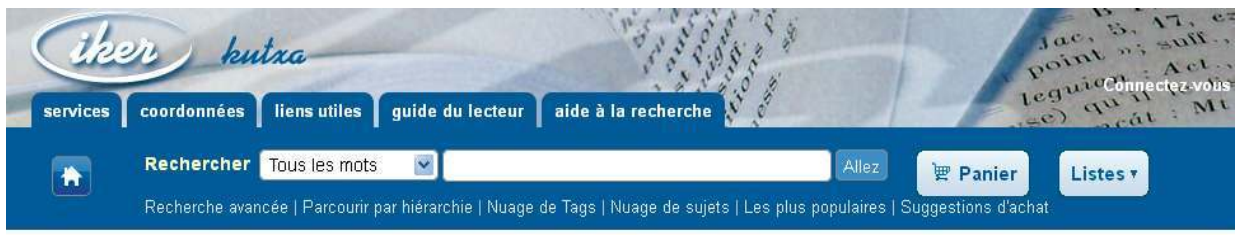

Bienvenue sur le catalogue documentaire

Le catalogue informatisé KUTXA regroupe les notices documentaires des livres, revues, thèses et mémoires disponibles au centre de recherche IKER UMR5478 (CNRS, Bordeaux 3. UPPA). Certains documents sont dématérialisés et accessibles en ligne.

Le champ documentaire est consacré aux études basques et notamment à la langue et aux textes : sociologie, anthropologie, sociolinguistique, linguistique, syntaxe, sémantique, dialectologie, langue, littérature, histoire, géographie.

(Publié le 21/01/2011)

\section{Accès direct auX contenus de KUTXA}

Acquisitions récentes | Liste des périodiques | Liste des thèses | Documents sur la langue basque

D'autres listes de notices sont accessibles via le formulaire de recherche $->$ Listes

(Publié le 18/03/2009)

Support, assistance technique

Vous pouvez bénéficier d'une assistance. Si vous ne trouvez pas de réponse satisfaisante dans KUTXA nous sommes prêts à vous aider à trouver une solution.

Indiquez-nous aussi les anomalies du catalogue et les dysfonctionnements techniques. Nous adressons un exemplaire gratuit de la revue annuelle à caractère scientifique et consacrée aux études basques Lapurdum, à ceux et celles qui nous proposent des données bibliographiques, pour la qualité et la pertinence des informations.

Figure 2 : Page d'accueil du catalogue documentaire Kutxa

Tableau 6 : Offre de ressources numériques (2012)

\begin{tabular}{|l|l|}
\hline Type de ressource & Nom de la ressource \\
\hline Portails documentaires & BIBLIOSHS, SCD Bordeaux III, SCD UPPA, HABE \\
\hline Base bibliographique & Kutxa \\
\hline Archive ouverte & Artxiker \\
\hline Revue en ligne & Lapurdum \\
\hline Actualités scientifiques & web du laboratoire \\
\hline
\end{tabular}




\section{Conclusion}

La direction du laboratoire IKER voulait accroître sensiblement les ressources documentaires du laboratoire en fonction des besoins liés aux travaux de recherche et des possibilités offertes par les nouvelles technologies de l'information et de la communication en 2007. Après quelques temps, il s'avère que le centre de documentation du laboratoire est installé sur le campus de la Nive (UPPA), ouvert aux étudiants avancés (à partir du master), enseignants et chercheurs pendant l'année universitaire et représente une référence dans le domaine de la linguistique basque ${ }^{36}$ à Bayonne. Nous proposons en grande majorité un fonds contemporain avec des monographies et périodiques spécialisés dans un espace de travail réaménagé récemment avec de nouveaux mobiliers et matériels informatiques (BISBROUCK 2010 : 23). Le fonds des imprimés a enregistré un accroissement important. Nous sommes passés d'une collection de 800 ouvrages en 2007 à plus de 2.800 titres aujourd'hui. Le catalogue documentaire Kutxa comprend plus de 2.000 notices bibliographiques qui décrivent les documents de l'unité de recherche (livres, thèses, mémoires, revues, documents audiovisuels, partitions, manuscrits, ressources électroniques) avec parfois des liens vers le texte intégral des ressources (articles, mémoires). Il repose sur les standards du web 2.0 ce qui permet aux internautes de s'approprier les informations (création de bibliothèque personnelle, export de données bibliographiques) et d'interagir de façon simple sur les contenus (annotations sur les pages) dans un espace public ou privé (compte lecteur).

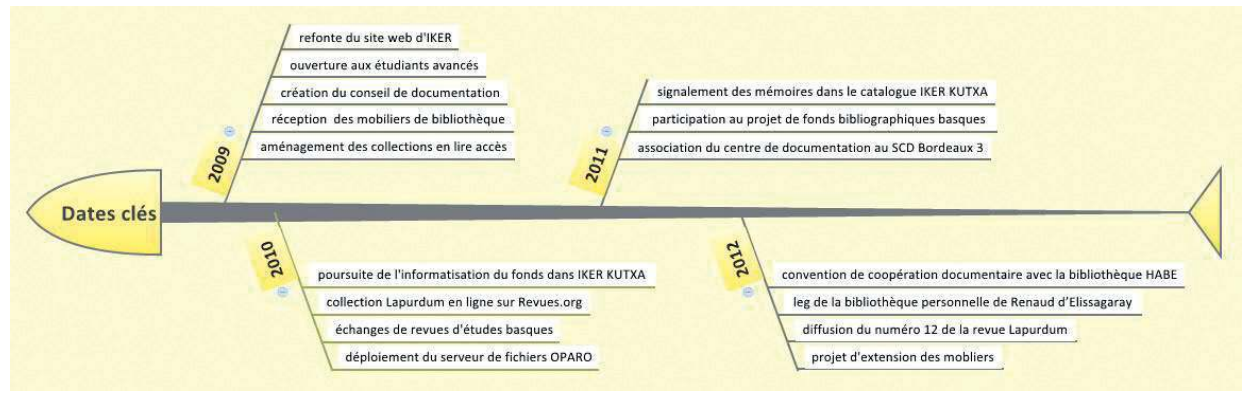

Figure 3 : Quelques dates clés sur la documentation

36.- Bibliothèques de l'UPPA sur la Côte basque : <http://bibliotheques.univ-pau.fr/live/bibliotheques/cote-basque> (consulté le ler septembre 2012) 
À l'origine du projet du centre de documentation, les questions qui se posaient étaient multiples. Il y avait des interrogations relatives au déménagement des collections et d'autres à l'organisation des accès à l'information dans les bâtiments du Château-Neuf de Bayonne. Il faut maintenant intégrer l'offre numérique (e-books et supports de lecture) dans la politique documentaire. La composition thématique des collections devrait être très largement marquée par des contenus linguistiques. Les prochaines activités devraient tourner autour de la formation des usagers aux ressources documentaires (DENECKER \& DURAND-BARTHEZ 2011. 69) et s'orienter aussi vers la pérennisation des données d'observation issues des projets de recherche du laboratoire dans une nouvelle bibliothèque numérique dont la mise en œuvre devra s'appuyer sur un logiciel libre (Spip, Lodel, Koha, Omeka, ...). Il semblerait enfin approprié d'activer le service Open Archives Initiative (OAI) du catalogue documentaire Kutxa pour que les métadonnées soient moissonnées par des moteurs de recherche fédérée (Babord $+^{37}$, Isidore ${ }^{38}$, Uztartuz, fonds bibliographique basque).

\section{Bibliographie}

\section{Décret}

Décret $n^{\circ}$ 2011-996 du 23 août 2011 relatif aux bibliothèques et autres structures de documentation des établissements d'enseignement supérieur créées sous forme de services communs. Paris: JORF no 0196, 25 août 2011.

\section{Articles}

BLIN, Isabelle \& OURET, Marie-Andrée. Mettre en réseau et valoriser les fonds documentaires basques : le projet d'un territoire. Lettres E Images d'Aquitaine, 2011, vol. 92, p. 7. Disponible sur : <http://ecla.aquitaine.fr/Ecla/Lettres-et-Images-d-Aquitaine/Articles/ Mettre-en-reseau-et-valoriser-les-fonds-documentaires-basques-Le-projet-d-un-territoire> (consulté le 1 septembre 2012)

COYOS, Jean-Baptiste. Artxiker: l'Archive de la Recherche pour la Langue basque et les Langues typologiquement proches. Lapurdum, 2010, vol. 10, pp. 343-349. Disponible sur : <http://lapurdum.revues.org/201> (consulté le 1 septembre 2012)

LE NAIL, Jacqueline. Langues régionales et bibliothèques. BBF, 2007, vol. 52, no. 3, pp. 3645. Disponible sur : <http://bbf.enssib.fr/consulter/bbf-2007-03-0036-006.pdf> (consulté le 1 septembre 2012)

LUZURIAGA, Gerardo. Liburutegi digitalak. Jakin, 2007, vol. 160, pp. 11-46.

LUZURIAGA, Gerardo \& KERETXEA, Roberto. Euskal liburutegi dugitalak : 2007-2009.

37.- PRES Département TIC. Babord+ : Base Documentaire des Universités de Bordeaux [en ligne]. Université de Bordeaux. Disponible sur : <http://www.univ-bordeaux.fr/babordplus> (consulté le 15 septembre 2012)

38.- Très Grand Equipement (TGE) Adonis. Isidore, plateforme de recherches en sciences humaines et sociales (SHS) [en ligne]. Disponible sur : <http://www.rechercheisidore.fr> (consulté le ler septembre 2012) 
Jakin, 2009, vol. 172, pp. 11-46.

POITELON, Jean-Claude. Préface de la bibliographie des publications périodiques du Pays basque parues en France de 1944 à 2004. Lapurdum, 2008, vol. 12, pp. 7-8. Disponible sur : <http://lapurdum.revues.org/1637> (consulté le 1 septembre 2012)

RENOULT, Daniel. Bibliothèques de recherche et mondialisation. In : La mondialisation de la recherche. Paris : Collège de France (Conférences), 2011. Disponible sur : <http:// conferences-cdf.revues.org/305> (consulté le ler septembre 2012)

RUIZ DE GAUNA LUCAS, Adolfo. Catálogo de publicaciones periódicas vascas existentes en Archivos, Bibliotecas y Hemerotecas del País Vasco. Método y problemas para su elaboración. In : Congresos de Estudios Vascos Xème Congres d'Etudes Basques: Pamplona 1987. Archives, Bibliothèques et Musées. Donostia: Eusko Ikaskuntza, 1988. no. 10. p. 579-586. Disponible sur : <http://www.euskomedia.org/PDFAnlt/congresos/10/10579586. pdf> (consulté le ler septembre 2012)

UGALDE, M. \& ETCHEVERRIA K.. Mendigainera: Scaling the peak of the Basque library backlog. Library resources E technical services, 1998, vol. 42, no. 4, pp. 304-312.

URQUIJO IBARRA, Julio de. Notas de Bibliografía vasca. 4, ¿ De qué obra francesa tradujo Duhalde sus Meditaciones ? Revista Internacional de los Estudios Vascos = Revue Internationale des Etudes Basques. 1908, no 2. pp. 313-323. [en ligne]. Disponible sur : <http://hedatuz.euskomedia.org/4768/1/02313323.pdf> (consulté le ler septembre 2012)

YANG, S. \& HOFMANN, M.. The Next Generation Library Catalog : A Comparative Study of the OPACs of Koha, Evergreen, and Voyager. Information Technology \& Libraries, 2010, vol. 29, no. 3, pp. 141-150.

\section{Livres}

AGIRREAZALDEGI BERRIOZABAL, Teresa (Ed.). Dokumentazioa eta ondare bibliografikoaren komunikazioa $=$ Documentación y comunicación del patrimonio bibliográfico. Bilbao : Universidad del País Vasco, Servicio Editorial = Euskal Herriko Unibertsitatea, Argitalpen Zerbitzua, 2011. 180 p.

AGIRREAZALDEGI BERRIOZABAL, Teresa (Ed.). Dokumentazioa eta eduki digitalen kudeaketa [Documentación y gestión de los contenidos digitales]. Bilbo : Euskal Herriko Unibertsitatea, Argitalpen Zerbitzua [Universidad del País Vasco, Servicio Editorial], 2010. $222 \mathrm{p}$.

AGIRREAZALDEGI BERRIOZABAL, Teresa (Ed.). Informaziorako dokumentazioaren oinarriak. Bilbo : Udako Euskal Unibertsitatea, 2006. 262 p. Disponible sur : <http://ueu. org/download/liburua/InformaziorakoDoku.osoa.pdf> (consulté le 1 septembre 2012)

BISBROUCK, Marie-Françoise. Bibliothèques d'aujourd'hui : à la conquête de nouveaux espaces. Paris : Éd. du Cercle de la librairie, 2010. 394 p.

BOËTSCH, Gilles (Ed.). Rapport de conjoncture du Comité national de la recherche scientifique. Paris : CNRS Éditions, 2011. 976 p.

CAILlOT, Patrice. Bibliographie de la presse française politique et d’information générale, des origines à 1944 : 64, Pyrénées-Atlantiques (anciennement Basses-Pyrénées). Paris : 
Bibliothèque nationale de France, Département des périodiques, 2002. 152 p.

CHARTIER, Roger (Ed.). Pratiques de la lecture. Paris : Payot, 2003. 323 p. (Petite bibliothèque Payot; 167)

DENECKER, Claire \& DURAND-BARTHEZ, Manuel. La formation des doctorants à l'information scientifique et technique. Villeurbanne: Presses de l'ENSSIB, 2011. 215 p.

ENGARD, Nicole. Pratical open source software for librairies. Oxford : Chandos Publishing, 2010. 200 p.

Izpegi, Comité (Ed.). La politique du patrimoine en Pays basque : état des lieux et perspectives: colloque du 25 mai 1991 organisé par le Comité Izpegi. Saint-Etienne-de-Baïgorry : Editions Izpegi, 1982. 283 p. (Recherches; ISSN 1285-9842)

MKADMI, Abderrazak \& SALEH, Imad. Bibliothèque numérique et recherche d'informations. Paris : Hermès Science publications \& Lavoisier, 2008. 280 p.

TORREALDAI, Joan Mari. 30 urte liburugintzan, 1976-2005. Donostia : Jakin, 2007. 182 p. (Jakin beha; 1)

\section{Sites web}

Centre pour l'édition électronique ouverte (Cléo) du CNRS. Calenda : Calendrier des sciences humaines et sociales [en ligne]. Disponible sur : <http://calenda.revues.org> (consulté le ler septembre 2012)

Centre pour l'édition électronique ouverte (Cléo) du CNRS. Lodel, logiciel d'édition électronique [en ligne]. Disponible sur : <http://www.lodel.org> (consulté le ler septembre 2012)

Centre pour l'édition électronique ouverte (Cléo) du CNRS. Revues.org - Portail de revues en sciences humaines et sociales [en ligne]. Disponible sur : <http://www.revues.org> (consulté le ler septembre 2012)

HABE IKASBIL. HABE Liburutegia [en ligne]. Disponible sur : <http://www.ikasbil.net/web/ liburutegia/home> (consulté le ler septembre 2012)

IKER UMR 5478 (CNRS, Bordeaux 3, UPPA) \& CCSD (Centre pour la Communication Scientifique Directe - UPS2275). Artxiker: Archive de la Recherche pour la Langue basque et les Langues typologiquement proches [en ligne]. Disponible sur : <http://artxiker.ccsd. cnrs.fr> (consulté le ler septembre 2012)

IKER UMR5478 (CNRS, Bordeaux 3, UPPA). Lapurdum - Euskal ikerketen aldizkaria Revue d'études basques [en ligne]. Disponible sur : <http://lapurdum.revues.org> (consulté le ler septembre 2012)

IKER UMR5478 (CNRS, Bordeaux 3, UPPA). Documentation. À propos du centre de documentation [en ligne]. Disponible sur : <http://www.iker.cnrs.fr/-centre-dedocumentation-.html> (consulté le ler septembre 2012)

IKER UMR5478 (CNRS, Bordeaux 3, UPPA). Documentation. Catalogue Kutxa [en ligne]. Disponible sur : <http://iker-koha.univ-pau.fr> (consulté le ler septembre 2012)

INIST-CNRS. INIST-CNRS. Présentation du portail BiblioSHS : portail d'information scientifique des unités CNRS en Sciences humaines et sociales [en ligne]. Nancy : (présentation). 
Disponible sur : <http://biblioshs.inist.fr/sites/biblioshs/IMG/ppt/TutorielBBSHSV3.pps> (consulté le ler septembre 2012)

KohaLa. koha-fr : communauté francophone koha [en ligne]. Disponible sur : <http://www. koha-fr.org> (consulté le ler septembre 2012)

Médiathèque. Mairie de Bayonne. Accueil du site de la médiathèque de Bayonne [en ligne]. Disponible sur : <http://www.mediatheque.bayonne.fr> (consulté le ler septembre 2012)

Musée Basque et de l'histoire de Bayonne. Bibliothèque et centre de documentation [en ligne]. Disponible sur : <http://www.museebasque.com/fr/documentation> (consulté le ler septembre 2012)

PRES Département TIC. Babord+ : Base Documentaire des Universités de Bordeaux [en ligne]. Université de Bordeaux, 2011. Disponible sur : <http://www.univ-bordeaux.fr/babordplus> (consulté le ler septembre 2012)

Très Grand Equipement (TGE) Adonis. Isidore, plateforme de recherches en sciences humaines et sociales (SHS) [en ligne]. Disponible sur : <http://www.rechercheisidore.fr> (consulté le ler septembre 2012)

Université Michel de Montaigne - Bordeaux 3, SCD. Accueil Service Commun de Documentation de l'Université Bordeaux 3 [en ligne]. Disponible sur : <http://www.u-bordeaux3.fr/fr/ documentation.html> (consulté le ler septembre 2012)

Université de Pau et des Pays de l'Adour (UPPA). Centre de ressources informatiques de l'UPPA [en ligne]. Disponible sur : <http://cri.univ-pau.fr/live> (consulté le ler septembre 2012)

Université de Pau et des Pays de l'Adour (UPPA), SCD. Accueil Service Commun de Documentation [en ligne]. Disponible sur : <http://bibliotheques.univ-pau.fr/live> (consulté le ler septembre 2012) 


\section{Annexe}

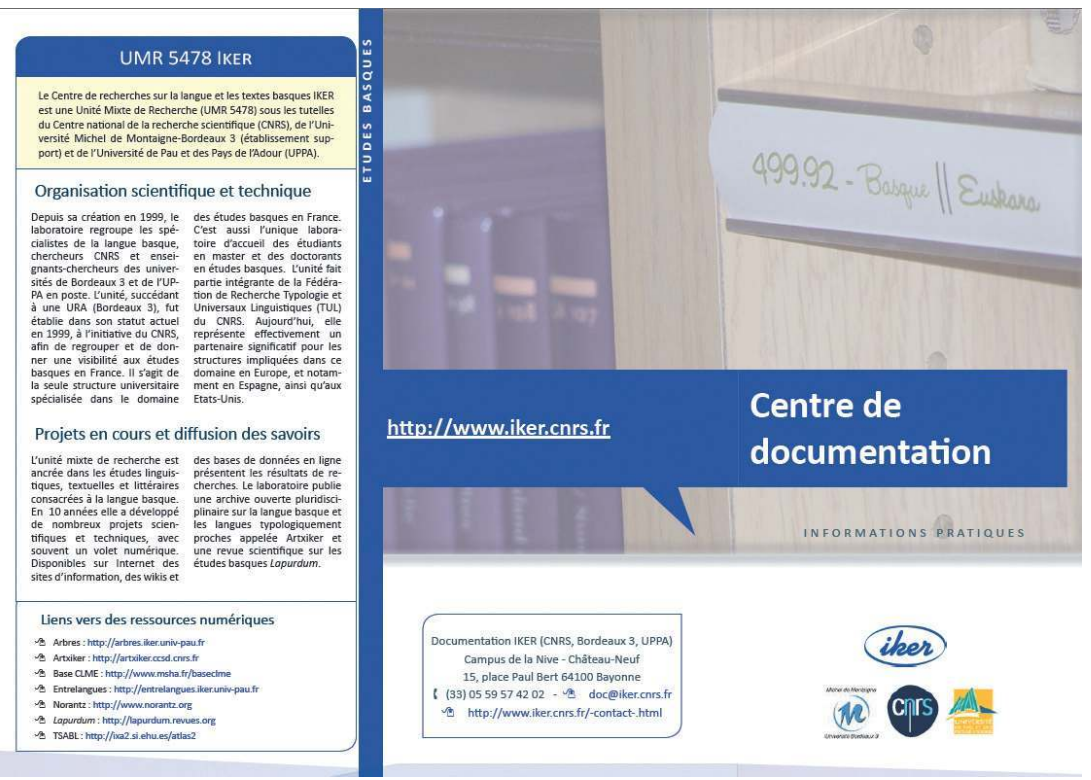

Annexe 1 : Plaquette de présentation du centre de documentation en français

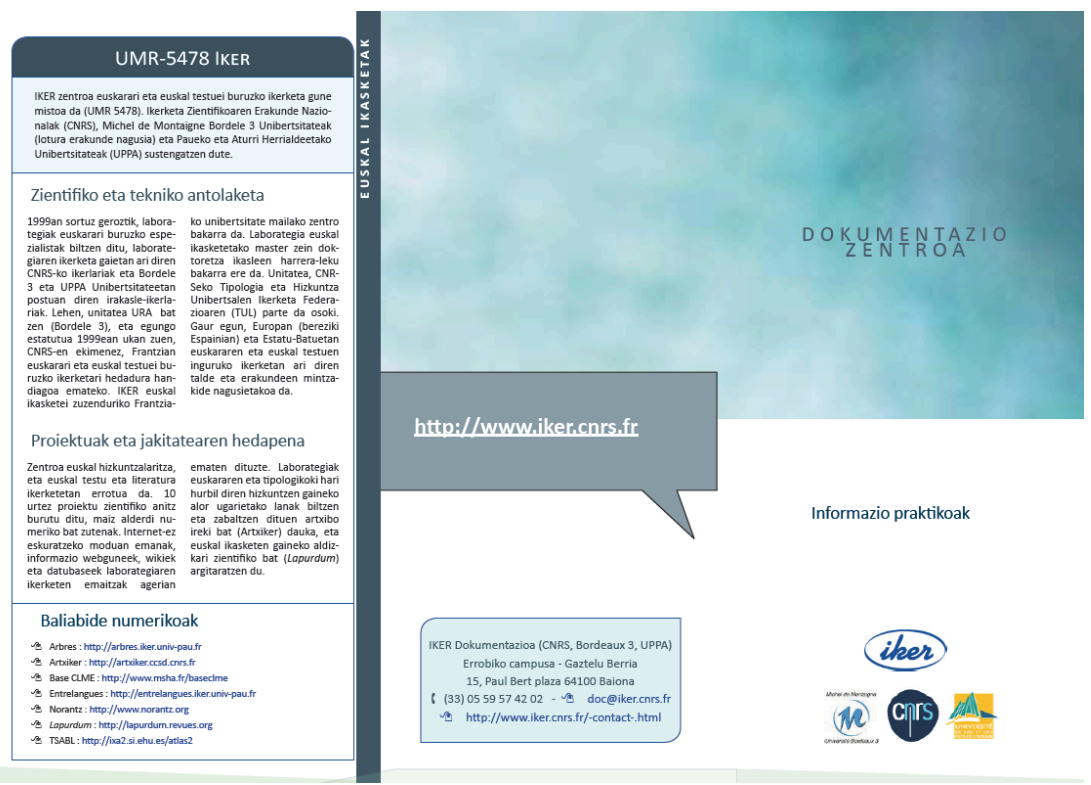

Annexe 2 : Plaquette de présentation du centre de documentation en basque 